\title{
RECHERCHES THÉORIQUES SUR L'ÉCOULEMENT DE COUCHES SUPERPOSÉES DE FLUIDES DE DENSITÉS DIFFÉRENTES "
}

\author{
THEORITICAL RESEARCH ON THE FLOW OF NON-HOMOGENOUS FLUIDS \\ par A. CRAYA \\ Ancien Elève de l'Ecole Polytechnique \\ Ingénieur aux Etablissements NEYRPIC \\ English Synopsis p. 7
}

Considérons un fluide cu repos présentant une stratification stable de densité; si l'on aspire au sein de ce fluide par un orifice, l'écoulement, contrairement à ce qui se passe pour les fluides homogènes constitue un fleuve interne dont la hauteur dépend du débit aspiré, de la répartition des densités, etc...

Ce problème s'est trouvé posé dans le projet d'utilisation de l'énergie thermique des mers tropicales; du fait des variations de température et de salinité la densité y croît avec la profondeur; il importait de connaître dans quelle mesure cette circonstance favoriserait l'aspiration d'eau froide en profondeur, en empêchant les couches supérieures chaudes d'être intéressées par l'écoulement; on rencontre également des problèmes connexes à propos de l'évacuation des eaux troubles des barrages, en météorologie, etc...

Les recherches théoriques exposées dans ces lignes ont été menées en liaison avec l'étude expérimentale faisant l'objet de la communication de M. Paul GARIEL.

\section{L'ASPIRATION LIMITE}

Le cas simple d'ailleurs fondamental qui nous occupera principalement sera celui de deux couches superposées de densité $+\Delta$ o et s'étendant indéfiniment dans le sens vertical, et limitées par une paroi verticale percée d'un orifice situé à une hauteur h au-dessus de l'interface (fig. 1)

Lorsque le débit aspiré est suffisamment fort les deux liquides participent à l'écoulement; si l'on diminue progressivement l'appel de débit, l'expérience montre qu'à partir d'un certain moment la quantité aspirée du liquide le plus dense devient très faible.

Il est vraisemblable que ce débit résiduel de liquide inférieur, ainsi que la formation d'une couche intermédiaire qui apparaît alors (2) sont des conséquences de la miscibilité des liquides; schématisant les résultats expérimentaux nous admettrons qu'en dessous d'un certain débit, ou au-delà d'une certaine houteur $h$, que nous appellerons conditions limites d'aspiration, le liquide inférieur ne participe rigoureusement plus à l'écoulement; notre analyse s'appliquera donc le plus parfaitement au cas de deux fluides non miscibles.

La recherche des lois de I'aspiration limite fera l'objet principal de cette étude (2).

\section{FACTEURS PHYSIQUES FONDAMENTAUX}

Qualitativement le mécanisme du phénomène se présente comme suit.

Au voisinage de l'orifice il règne un appel des particules liquides; d'autre part une particule du liquide le plus dense supposée isolée au milieu du liquide plus léger subit une poussée d'Archimède tendant à la faire couler vers son lieu d'origine

L'équilibre de l'interface apparaît alors comme un compromis entre l'aspiration qui l'attire vers l'orifice et la pesanteur qui tend à l'étaler.

Les paramètres fondamentaux de l'aspiration limite qui résultent de cette analyse sont des lors les suivants:

Densités $0+\triangle p$ et 0 des deux liquides.

Débit limite : débit linéaire q si l'on considère un écoulement plan à travers une fente; débit total $Q$ s'il s'agit d'un écoulement à trois dimensions.

(1) Communication au V/o Congrès International de Mécanique Appliquée (Septembre 1946, Paris).

(2) Cf. Compte rendu à l'Académie des Sciences, séance du 25 mars 1946 T. 222 , page 1159. 
Hauteur limite h.

Accélération $\mathrm{g}$ de la pesanteur.

Ce dénombrement des variables est-il bien complet et n'en avons-nous pas omis d'essentielles?

II peut sembler notamment au premier abord, que les dimensions de l'orifice doivent jouer un certain rôle; en réalité on sait que l'aspiration produite par un orifice est sensiblement indépendante de ses dimensions, dès qu'on se place suffisamment loin de celui-ci; elle équivaut alors pratiquement à celle d'un puits ponctuel; nous nous réservons d'ailleurs de revenir ultérieurement sur ce point et de préciser les conditions de validité de notre hypothèse.

On peut aussi suspecter la viscosité d'avoir quelque influence; elle intervient, en effet, mais plutôt, semble-t-il, par ses effets cumu!ès sur un fleuve sous-marin à grande distance de l'orifice que sur son comportement au voisinage immédiat de celui-ci ; c'est également un point que nous reprendrons en détail ultérieurement.

La tension interfaciale est enfin un facteur qui peut dans certains cas entrer en compte mais que nous considérerons ici comme secondaire: elle explique notamment que dans certaines expériences les choses ne se passent pas de même suivant qu'on opère en augmentant ou diminuant le débit.

On conçoit enfin, que notre schématisation consistant à supposer les deux liquides indéfinis vers le haut et vers le bas n'a rien d'essentiel, et que la présence d'une surface libre ou d'un fond n'altère pas sensiblement les résultats pourvu qu'ils soient situés suffisamment loin de l'interface.

En résumé nous faisons jouer le rôle principal dans le phénomène qui nous occupe au jeu de l'inertie et de la pesanteur au voisinage de I'orifice.

\section{APPLICATION DE L'ANALYSE DIMENSIONNELLE}

Si nous demandons à la Mécanique des Fluides une théorie de l'aspiration limite, celle-ci se traduira dans ces conditions par une relation:

$$
f(q, h, g, p, \Delta p)=0
$$

Nous pouvons maintenant apporter d̀ ce qui précède une précision très intéressante.

Considérons pour cela de plus près les condi- tions d'équilibre de l'interface (dont "y " désignera l'ordonnée).

Du côté du liquide en mouvement le théorème de Bernoulli donne :

$$
p+\frac{V^{2}}{2}+g y=C^{t e}
$$

Du côté du liquide le plus dense au repos on a en vertu de l'équilibre hydrostatique

$$
p+(p+\Delta p) g y=C^{\text {te }}
$$

On en déduit par différence :

$$
\frac{V^{2}}{2}=\frac{\Delta p}{p} g y+C^{\text {te }}
$$

On voit que I'on peut faire abstraction du liquide inférieur et considérer l'interface comme une surface libre sous l'action d'une pesanteur changée de sens et réduite à $\frac{\Delta p}{p} g$

Quant à l'écoulement du liquide supérieur il s'agit d'un champ à potentiel de vitesse, dont les particularités dépendent uniquement de la géométrie des parois fixes, du débit, et pour ce qui est de l'interface, du paramètre $\stackrel{\Delta p}{-g}$ et de la hauteur $h$ fixant la constante de la relation imposée 3 - 2.

En résumé les précisions qui précèdent conduisent à affirmer que dans la relation $3-1$ les quantités $g$, $p$ et $\Delta \rho$ doivent se trouver bloquées en un paramètre unique $\frac{\Delta_{\hat{i}}}{\hat{i}} \mathrm{~g}$ et que celle-ci s'écrit dès lors

$$
p\left(q, h, \frac{\Delta \rho}{p} g\right)=0
$$

Cette équation devant être l'aboutissement d'une théorie que nous venons de reconnaître purement cinématique, doit être homogène par rapport aux deux grandeurs cinématiques fondamentales qui sont la longueur et le temps; comme elle comporte trois termes il en résulte en vertu du théorème des $\Pi$, qu'elle doit se réduire à une fonction d'un seul paramètre $\|$ de dimensions nulles et s'écrire par conséquent $I I=C^{\text {te }}$.

11 est facile de former ce paramètre et nous distinguerons pour cela deux cas. 
S'il s'agit d'un écoulement plan à travers une fente de débit linéaire $q$ on aura :

$$
\mathrm{I}=\frac{\Delta p}{\rho} \mathrm{g} \frac{h^{3}}{\mathrm{q}^{2}}=\mathrm{C}^{\mathrm{te}}
$$

S'il s'agit d'un écoulement à trois dimensions à travers un orifice pratiquement ponctuel de débit total $Q$ il faut prendre:

$$
\Pi=\frac{\Delta \theta}{Q} \mathrm{~g} \frac{h^{3}}{Q^{2}}=C^{t e}
$$

Nous sommes donc assurés avant tout calcul, comme conséquence des hypothèses physiques émises, que les lois de l'aspiration limite doivent avoir la forme qui précède; ce résultat est évidemment très intéressant et il tient à ce que nous nous trouvons ici dans les conditions les plus favorables de l'Analyse dimensionnelle, celui où le nombre des grandeurs qui interviennent dépasse seulement d'une unité celui des grandeurs fondamentales.

II arrive que les succès de l'Analyse dimensionnelle parce qu'ils sont en apparence faciles paraissent suspects; ce sentiment n'est pas justifié, si l'on n'oublie pas que suivant le mot de Bridgman elle constitue "I'Analyse d'une Analyse" et qu'elle doit donc toujours trouver place entre l'examen physique du phénomène étudié et le calcul effectif de sa loi.

\section{THEORIE ELEMENTAIRE}

L'étape consécutive qui consiste dans le cas présent à calculer la valeur des constantes des formules $3-4$ et $3-4^{\prime}$ représente un problème assez difficile.

Cela tient même en se bornant aux champs plans (ce que nous ferons dans les paragraphes 4,5 et 6 ) à la forme $3-2$ de la condition imposée à l'interface; on connaît très peu de solutions rigoureuses de tels champs, lorsque les parois fixes sont assignées à l'avance.

Nous nous en tiendrons donc dans ce qui suit à des solutions approchées, et pour commencer nous voudrions donner un schéma théorique rudimentaire sans doute, mais ayant l'avantage de donner une image assez parlante du phénomène et un ordre de grandeur de la constante de la formule 3 - 4 .

Nous allons chercher pour cela dans quelles conditions il est impossible de trouver un point de raccordement $B$ de l'interface sur la paroi PB (fig. 1).

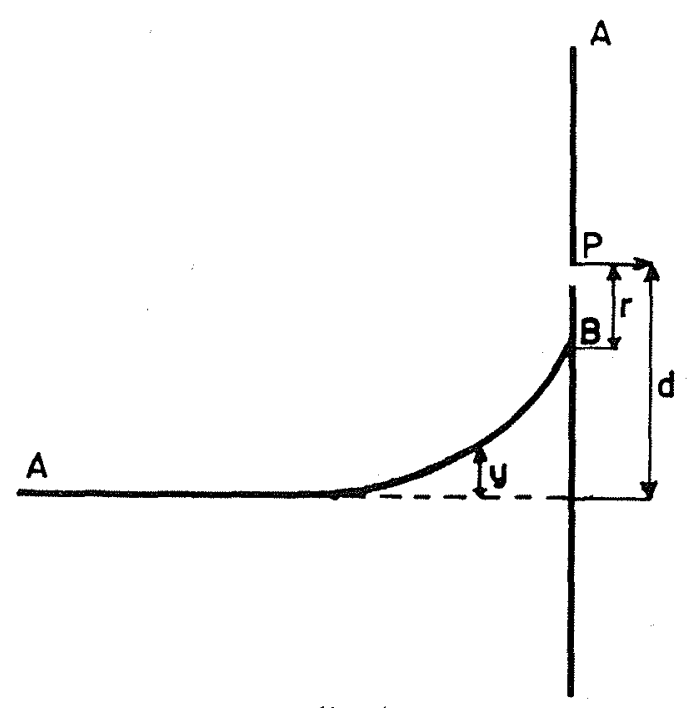

fig. 1

La vitesse en $B$ vérifie la condition imposée par l'interface :

$$
\frac{V_{B}{ }^{2}}{2}=\frac{\Delta p}{p} g(d-r)
$$

D'autre part la répartition des vitesses le long de $P B$ tend ou voisinage de $P$ vers celle $d^{\prime}$ un point puits

$$
V=\frac{\mathrm{q}}{\pi r}
$$

Nous admettrons - et c'est en ce point que notre schéma est grossier - que cette répartition vaut encore au voisinage de $B$.

La position du point de contact B est alors donnée par la relation :

$$
\frac{V_{B}^{2}}{2}=\frac{\Delta \rho}{\rho} g(d-r)=\frac{q^{2}}{2 \pi^{2} r^{2}}
$$

La figure (2) représente les deux expressions de $V^{2} / 2$ dont l'une est une droite (D) et l'autre une courbe (B) d'allure hyperbolique.

Si par exemple maintenant le débit q constant, on diminue progressivement la distance $d$, la droite (D) se déplace parallèlement à elle-même et à partir d'une certaine distance $h$ il n'y a plus de point de contact possible.

Les conditions d'aspiration limite correspondraient d'après cela au moment où (D) est tongente à (B), c'est-à-dire où l'équation $4-3$ a une racine double, ce qui exige:

$$
r=\frac{2 d}{3} \quad \Pi=\frac{\Delta \rho}{\rho} \frac{g h^{3}}{q^{2}}=\frac{27}{8 \pi^{2}}=0,342 \quad(4-4)
$$




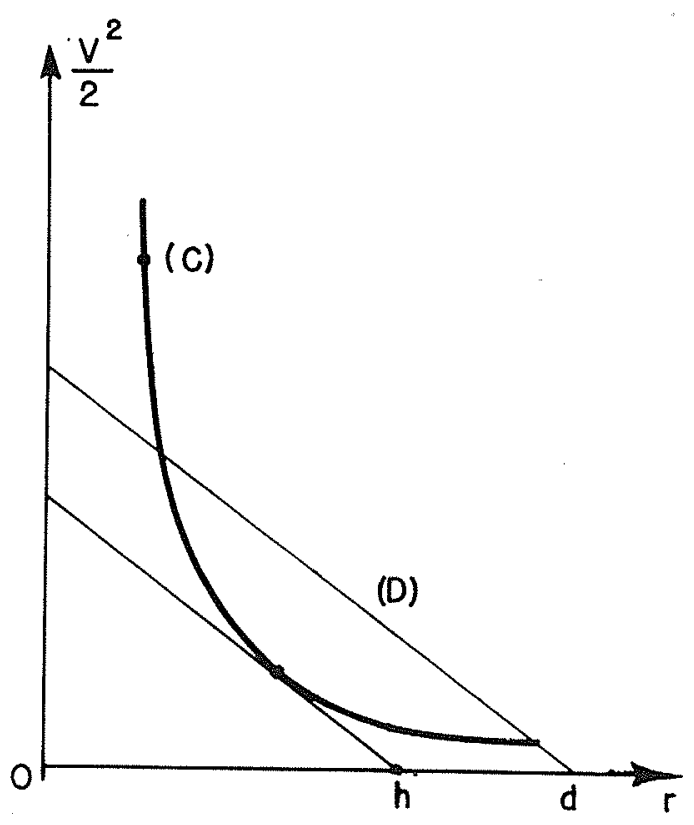

fig. 2

Le point faible de ce raisonnement est comme nous l'avons noté que la proximité de l'interface modifie le champ de vitesses 4 - 2 .

Si nous posons plus généralement pour le point de contact $B$

$$
V_{B}=k \frac{q}{\pi r}
$$

et si nous appelons $\mathrm{m}$ le rapport $\mathrm{r} / \mathrm{h}$ fixant la position de B pour l'aspiration limite, nous aurons :

$$
\frac{V_{B}^{2}}{2}=\frac{\Delta \rho}{\rho} g h(1-m)=k^{2} \frac{q^{2}}{2 \pi^{2} m^{2} h^{2}}
$$

soit

$$
\Pi=\frac{\Delta \rho}{\rho} \quad \frac{g h^{3}}{q^{2}}=\frac{k^{2}}{2 \pi^{2} m^{2}(1-m)}
$$

La formule 4-5 montre comment la constante de la loi cherchée se relie aux éléments cinématiques inconnus du problème; l'évaluation 4 - 4 revient à prendre pour le numérateur et le dénominateur les plus grandes valeurs possibles; il y a par là une certaine compensation d'erreur qui explique le succès de cette estimation vis-à-vis de l'expérience et d'un calcul ultérieur plus complet; mais pour ce qui est du détail, l'étude de divers champs plans montre que $k$ est inférieur à l'unité et plus proche de
$1 / 2$ et que la valeur $m==2 / 3$ correspond à une position de $B$ trop éloignée du point puits $P$.

\section{UN SCHEMA SIMPLIFIE}

Les remarques qui précèdent épuisent semblet-il ce qui peut être dit par voie élémentaire.

La solution complète doit être demandée à l'étude des champs avec interface vérifiant la condition 3-2: on peut cependant à titre préliminaire considérer un schéma approché conduisant à un problème plus simple.

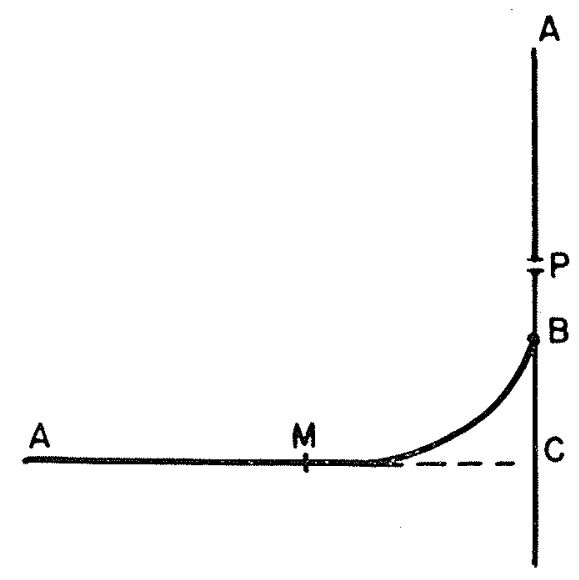

fig. ?

Pour cela nous remplacerons (fig. 3) l'interface exacte inconnue par une portion de paroi plane $A M$ suivie d'une ligne de jet $M B$ à vitesse constante " $a$ " et nous imposerons à cette vitesse d'avoir la valeur qui serait celle en B de l'interface réelle:

$$
\frac{a^{2}}{2}=\frac{\Delta \hat{r}}{p} \overline{C B}
$$

Autrement dit nous remplaçons la loi exacte 3 - 2 représentée par $A B$ (fig. 4) par une loi AMB.

L'odographe de ce champ (plus exactement du champ symétrique par rapport à $A M$ ) est représenté par la figure 5 a.

On peut de plusieurs façons transformer ce domaine dans un demi-plan ? (fig. 5), par exemple en passant par le champ $v=\log \frac{w}{-}$ (fig. 5 b). 


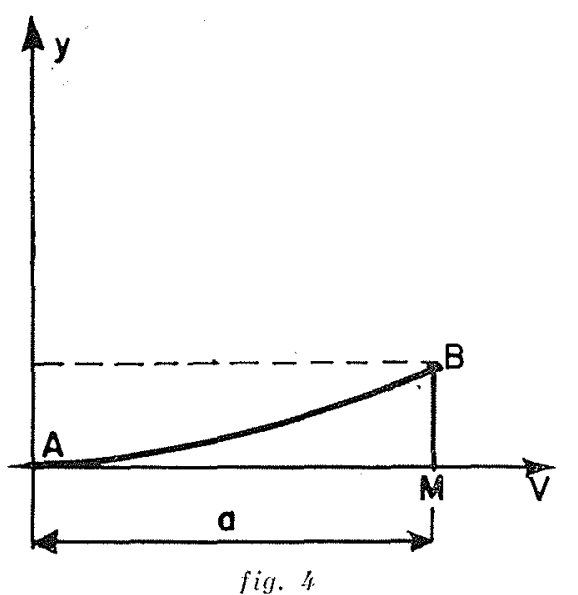

Lo transformation SCHWARTZ - CHRISTOFFEL. donne

$$
\frac{d v}{d \zeta}=k \frac{1}{\zeta-\%} \sqrt{\frac{\zeta}{\zeta+1}}
$$

dont l'intégration conduit en posant

$$
t=\sqrt{\frac{\zeta}{\zeta+1}}
$$

(plan représentatif fig. $5 d$ ) à :

$$
v=K \log \frac{t+1}{t-1}\left(\frac{t-m}{t+m}\right)^{m} \text {. où } m=\sqrt{\frac{a}{a+1}}
$$

Les proportions de figure du plan $u$ imposent ensuite $m=1 / 2$ et $K=i$, d'où:

$$
\frac{w}{a}=i \frac{t+1}{t-1} \sqrt{\frac{2 t-1}{2 t+1}}
$$

L'écoulement du plan $t$ (source en $A$, puits en $P$ de débit $q)$ est représenté par :

$$
z=\frac{q}{\pi} \log \frac{t^{2}-1 / 4}{t^{2}-1}
$$

La définition de l'odographe $w=\frac{d Z}{d z}$ conduit alors à :

$$
d z=\frac{6 q i}{\pi a} \frac{1}{4 t^{2}-1} \frac{t}{(t+1)^{2}} \sqrt{\frac{2 t+1}{2 t-1}} d t
$$

dont l'intégration s'effectue aisément en posant $u=\sqrt{\frac{2 t+1}{2 t-1}}($ plan représentatif fig. $5 \mathrm{e})$

$$
z=-\frac{q i}{\pi a}\left[\frac{2 u\left(u^{2}+1\right)}{3 u^{2}-1}-\frac{2}{\sqrt{3}} \log \frac{u \sqrt{3}+1}{u \sqrt{3}-1}\right]
$$

On en déduit la position des points caractéris. tiques de la figure 3 :

$$
\begin{aligned}
P C & =0,932 \frac{q}{\pi a} \\
P B & =0,452 \frac{q}{\pi a} \\
C M & =1,209 \frac{q}{\pi a}
\end{aligned}
$$

On remarquera que notre schéma impose le point de décollement $M$; si la paroi $A M$ se prolongeait jusqu'en $C$ it y aurait un maximum de vitesse (donc un minimum de pression) sur la paroi $A M$ en un point situé à la distance $C P$ de $C$; notre point $M$ ne s'en écarte pas beaucoup.

Si l'on s'imposait une autre distance $C M$, il $y$ curait un maximum de vitesse sur $A M$ pour $M$ plus voisin de $C$, et une inflexion de la ligne de jet pour $M$ plus éloigné ; l'odographe (fig. 5 a) comporterait alors une pointe en $M$ prolongeant soit $A M$ soit $B M$; ces champs seraient évidemment moins aptes à schématiser la vraie ligne de jet.

On voit que notre calcul conduit pour la vitesse du point de raccordement à la relation :

$$
a=k \frac{\mathrm{q}}{\pi \overline{\mathrm{PB}}} \operatorname{arec} \mathrm{k}=0,452
$$

et pour la position du point de raccordement à $\overline{\mathrm{PB}}$

$$
\frac{\overline{P C}}{\overline{P C}} \quad \mathrm{~m} \quad \text { avec } \mathrm{m}=0,485
$$

L'introduction de la condition $5-1$ ou ce qui revient au même la formule 4 - 5 donnent alors

$$
\Pi=\frac{\triangle \rho}{\rho} \frac{g h^{3}}{q^{2}}=a, 0856
$$

Cette valeur du paramètre II est quatre fois plus faible que celle de la théorie élémentaire, et comme la hauteur limite intervient dans 11 par son cube le présent schéma la réduit de $37 \%$ par rapport au calcul sommaire antérieur.

Cependant quoique mieux exploré le schéma actuel ne représente toujours qu'une approximation la valeur du paramètre II dépend beaucoup nous l'avons vu de la position du point de raccordement et celle-ci est sensible à la loi exacte de l'interface; nous en venons donc maintenant à la seule voie véritablement satisfaisante qui est l'étude des champs satisfaisant rigoureusement à la condition $3-2$. 


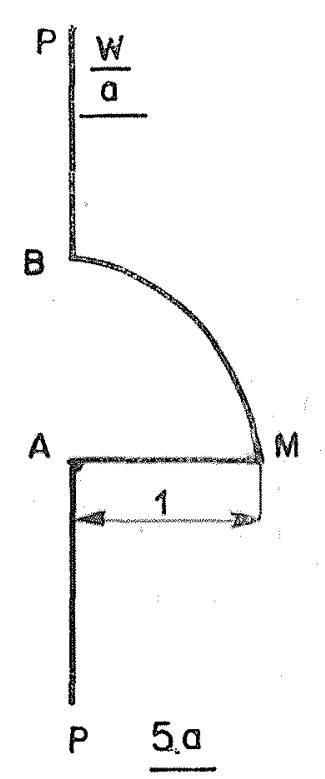

(ל)

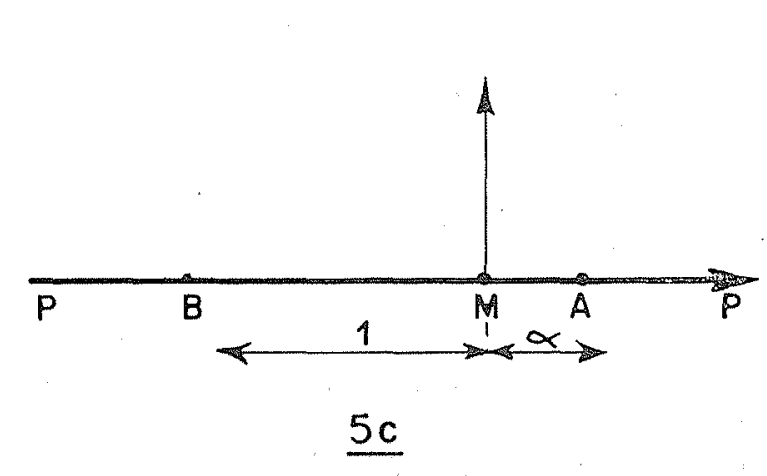

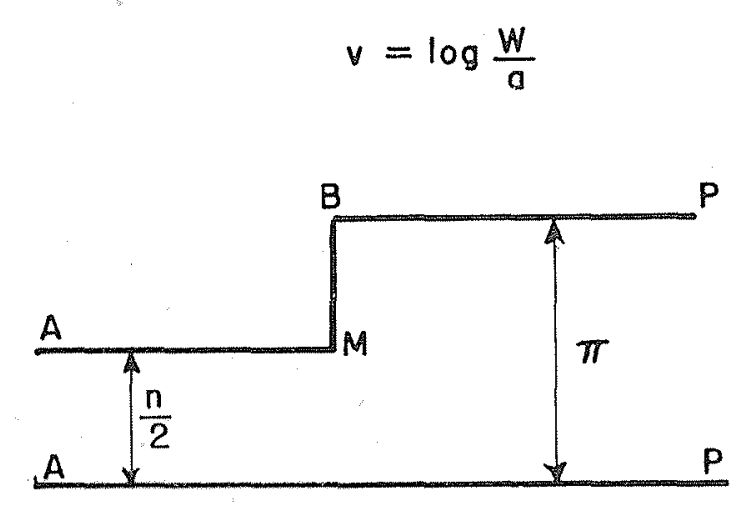

$5 b$

(1)

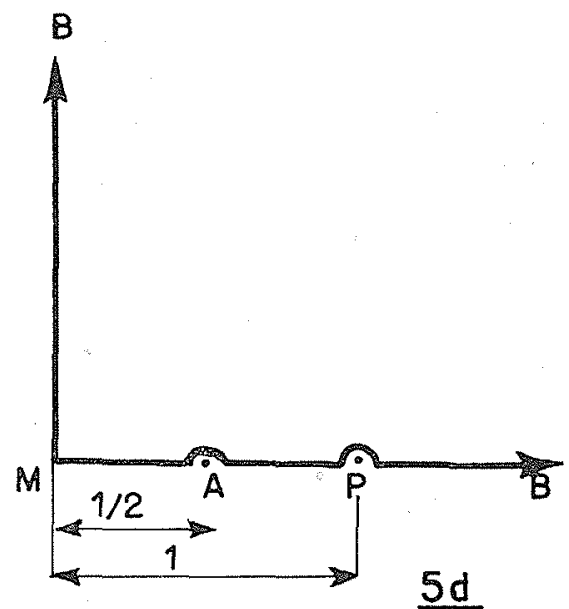

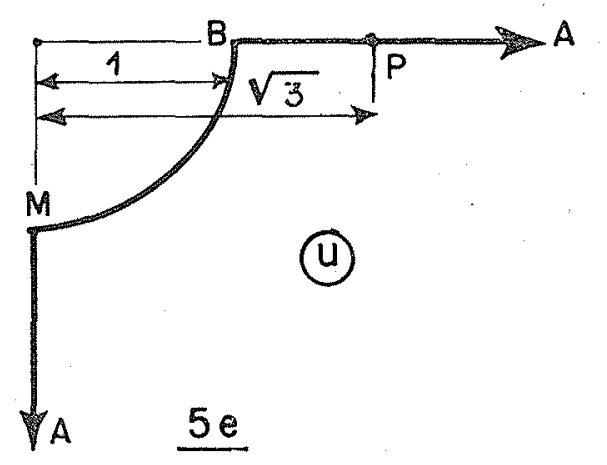

fig. 5 


\section{FORMATION D'UN CHAMP VERIFIANT LA LOI DE L'INTERFACE}

Nous utiliserons pour cela une méthode permettant de bâtir de tels champs, indiquée par A.-R. RICHARDSON (1).

Considérons une fonction analytique $Z(z)$ dont nous désignerons la dérivée par $w=\frac{d Z}{d z}$

Nous prendrons pour l'odographe une expression

$$
i w=\mu G^{1 / 3}\left[G^{\prime}+i \sqrt{1-G^{\prime 2}}\right] \quad(6-1)
$$

où la fonction $G(Z)$ sera astreinte à vérifier les conditions suivantes:

Sur une portion d'une ligne de courant $Z=X+i B\left(B=C^{t e}, X\right.$ variant entre deux valeurs déterminées) $G^{1 / 3}(Z)$ est supposé réel, $G^{\prime}(Z)$ est également réel et compris entre 0 et 1 .

11 est facile de voir que la ligne de courant correspondante du plan $\mathrm{z}=\mathrm{x}+\mathrm{i}$ y vérifie alors la relation :

$$
\frac{V^{2}}{2}=\frac{\mu^{3}}{3}\left(y-y_{0}\right)
$$

(où $V$ désigne la vitesse, c'est-à-dire $|w|$ ).

En effet, on a d'après l'équation $6-1$

$$
V^{2}=\mu^{2} G^{2 / 3}
$$

Cette même équation s'écrit d'autre part

$$
1
$$

$$
d z=\frac{1}{\mu G^{1 / 3}}\left[\sqrt{1-G^{\prime 2}}+i G^{\prime}\right] d Z
$$

On en déduit

$$
d y=\frac{1}{\mu} \frac{G^{\prime}}{G^{1 / 3}} d z \quad y-y_{0}=\frac{3}{2 \mu} G^{2 / 3}
$$

d'où la relation annoncée.

Pour que la ligne en question vérifie la condition fondamentale 3 - 2 de l'interface il suffira donc de prendre

$$
\mu^{3}=3 \frac{\Delta p}{p} g
$$

II s'agit maintenant de faire pour $G(Z)$ un choix assez heureux pour que les autres frontières du champ se rapprochent de celles qui nous intéressent; nous prendrons pour cela

$$
\mathrm{G}=\frac{2 \mathrm{q}^{\prime}}{\pi} \mathrm{e}^{\frac{\bar{\pi}}{2 \mathrm{q}^{\prime}} \mathrm{z}}
$$

(1) Phit. Mag., 1920, No 40.
Pour $Z$ réel variant de $-\infty$ à 0 on voit que $G^{\prime}(Z)$ est réel et varie de 0 à 1 ; ces valeurs de $Z$ donneront par conséquent une interface d'après les valeurs de la vitesse complexe donnée par 6 - 1, celle-ci aura la forme A B (fig. 6 f).

On voit aussi que lorsque $Z$ est encore réel mais varie de 0 à $+\infty$ on a dans le plan $z$ un bout de paroi rectiligne $B P$; enfin si l'on fait $Z=X+i q$ 'on obtient la ligne de courant A P rectiligne et inclinée de $30^{\circ}$ sur $\mathrm{P} \mathrm{x}^{\prime}$.

- La suite des figures 6 a à 6 e traduit de manière plus parlante les différentes fonctions intervenant dans le champ, on remarquera notamment que si l'on pose :

on en déduit

$$
v=\sqrt{1-G^{\prime 2}}-i G^{\prime}
$$

$$
G^{\prime}=\frac{1}{2}\left(i v+\frac{1}{i v}\right)
$$

de sorte que l'on passe d'un plan à l'autre par une transformation JOUKOWSKY (fig. 6 a et $6 c)$.

Nous venons donc d'obtenir par cette voie synthétique un champ qui s'il n'est pas exactement celui que nous cherchons s'en rapproche beaucoup ; d'autant que son prolongement analytique à travers AP s'obtient par simple symétrie et donne en particulier une ligne de courant $\mathrm{PA}_{1}$ (fig. $6 \mathrm{f}$ ); en définitive la seule différence avec l'écoulement proposé (fig. 3) réside dans cette paroi $\mathrm{PA}_{1}$ qui partie suivant la verticale se recourbe au lieu de demeurer rectiligne.

Il en résulte que l'homologue du débit que nous avons appelé q antérieurement, se relie à $q^{\prime}$ par

$$
\mathrm{q}^{\prime}=\frac{2}{3} \mathrm{q}
$$

Remplaçant dans 6-2 j., $G(Z)$ et $q^{\prime}$ par leurs expressions et posant

$$
e^{\frac{\pi}{2} \frac{z}{q}}=t \quad \text { et } \frac{1}{l}=\sqrt[3]{\frac{\pi^{2}}{2} \frac{\Delta p}{p} \cdot \frac{g}{q^{2}}}
$$

nous obtenons

$$
\frac{z}{i}=\int_{1}^{t}\left[\sqrt{\frac{1}{t^{3}}-1}+i\right] d t
$$




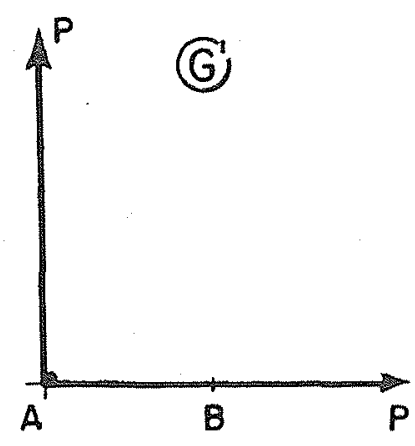

$6 a$

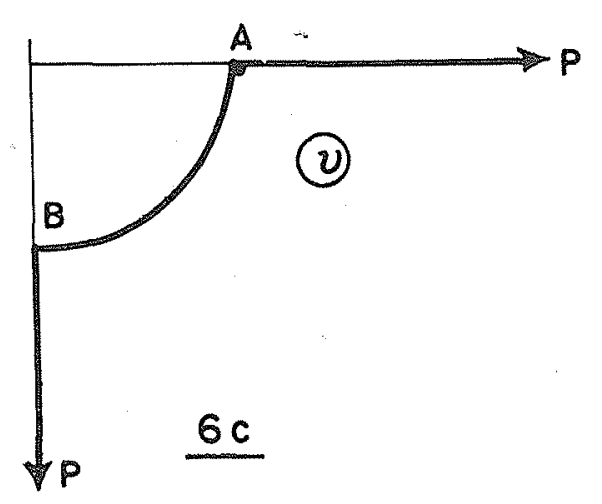

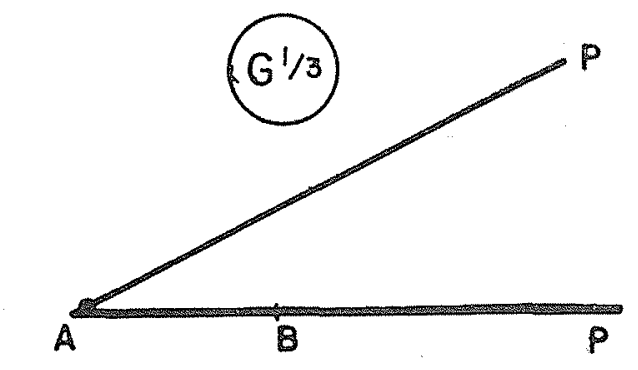

$\underline{6 d}$

(2)

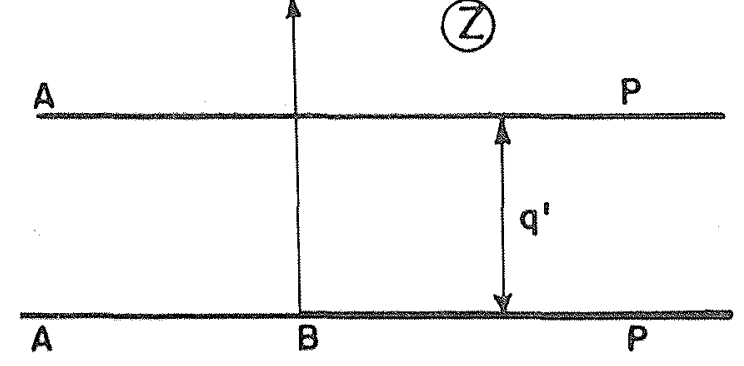

$\underline{6 b}$
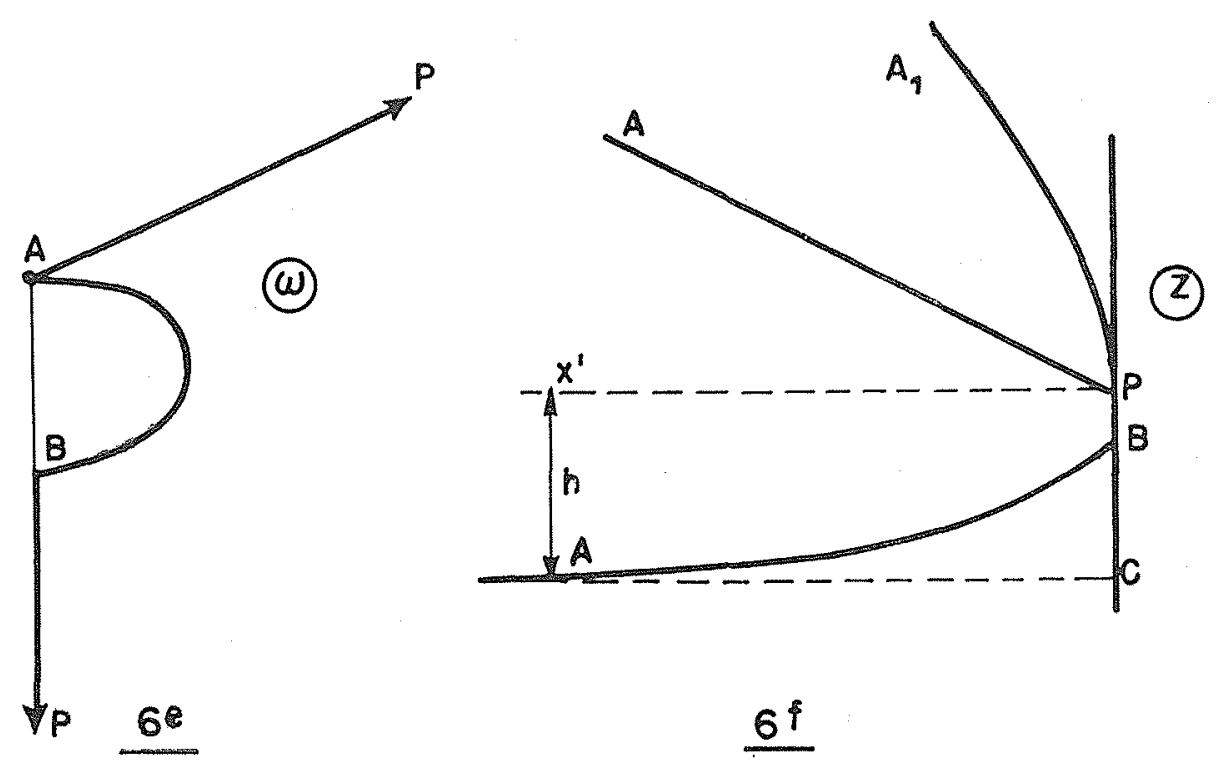

fig. 6 
On en déduit

$$
\begin{aligned}
& \overline{\mathrm{CB}}=l \\
& \overline{\mathrm{BP}}=l \int_{1}^{\alpha}\left[1-\sqrt{1-\frac{1}{t^{3}}}\right] \mathrm{dt}=0,293 \mathrm{i} \\
& h=1.293 l
\end{aligned}
$$

Par suite

$$
\|=\frac{\Delta \rho}{\rho} g \frac{h^{3}}{q^{2}}=0,438
$$

Nous tombons sur une valeur du paramètre ll assez proche de la première estimation sommaire (les hauteurs limites ne diffèrent que de $8 \%$ ) avec cependant des paramètres $k$ et $m$ de la formule 4 - 5 très différents :

$$
V_{B}=0,586 \frac{q}{\pi \cdot \overline{P B}} \text { et } \frac{\overline{\mathrm{PB}}}{\overline{\mathrm{CP}}}=m=0,226
$$

Il est enfin intéressant de calculer le profil de la surface libre qui s'écrit sous forme paramétrique (origine en $B$ )

$$
\left\{\begin{array}{l}
x=l \int_{1} \sqrt{t^{3}}-1 d t \quad 0<t<1 \\
y=l(t-1)
\end{array}\right.
$$

On peut par exemple effectuer ce calcul en se ramenant aux intégrales de LEGENDRE de $I^{\text {re }}$ et $2^{\circ}$ espèces; on obtient ainsi le tableau suivant: valeurs possibles du paramètreII, qui permettrait de définir en toute rigueur les conditions limites d'aspiration.

Nous n'insisterons pas davantage sur cette question, et nous nous proposons de revenir maintenant sur quelques points laissés en suspens au début de cette étude.

\section{INFLUENCE DES DIMENSIONS DE L'ORIFICE}

Nous avons dit que l'on peut considérer l'orifice comme ponctuel dès que ses dimensions sont suffisamment petites; nous voudrions préciser ce point.

Considérons pour cela le champ plan de la figure 7 ef faisons abstraction de la présence de l'interface.

Soit c la vitesse à la surface libre de la veine; on sait que la contraction du jet a pour valeur:

$$
\frac{1}{2 r_{0}}=\frac{\pi}{\pi+2}
$$

de sorte que

$$
c=\frac{q}{l}=\frac{\pi+2}{2 \pi} \frac{q}{r_{0}}
$$

Le champ de vitesse $V$ le long de la paro

\begin{tabular}{|c|c|c|c|c|c|c|c|c|}
\hline$-\frac{x}{l}$ & 0.014 & 0.105 & 0.337 & 0.766 & 1.488 & 2.738 & 5.262 & 12.818 \\
\hline$-\frac{y}{l}$ & 0.051 & 0.187 & 0.366 & 0.549 & 0.711 & 0.839 & 0.929 & 0.982 \\
\hline
\end{tabular}

La forme de cette courbe se rapproche assez de celles que révèle l'expérience: celle-ci a également confirmé la loi de similitude représentée par le paramètre II ainsi que sa valeur numérique $6-3$ (voir communication de $M$. Paul GARIEL).

Le problème semble donc suffisamment exploré pour les besoins de la pratique; du point de vue mathématique cependant une solution complète nécessiterait quelques prolongements; ce serait d'abord la formation d'un champ correspondant exactement à la fig. 1 (paroi verticale PA plane); ce serait surtout l'étude systématique de tous les champs correspondant aux est d'autre part donné en posant $t=V / c$ par :

$$
\frac{r}{r_{0}}=\frac{2}{\pi+2}\left[\frac{1}{t}+2 \operatorname{Arctg} t\right]
$$

On voit que le terme Arc tg t représente l'influence propre de la dimension de l'orifice; si on le néglige en effet l'équation $7-1$ se réduit à la loi du puits ponctuel; cette influence s'évanouit d'ailleurs très vite quand on s'éloigne de $P$ et elle est déjà faible pour un point distant de $P$ de la hauteur de la fente.

On peut s'en tenir à cette remarque et en conclure que les lois de l'aspiration limite ne 


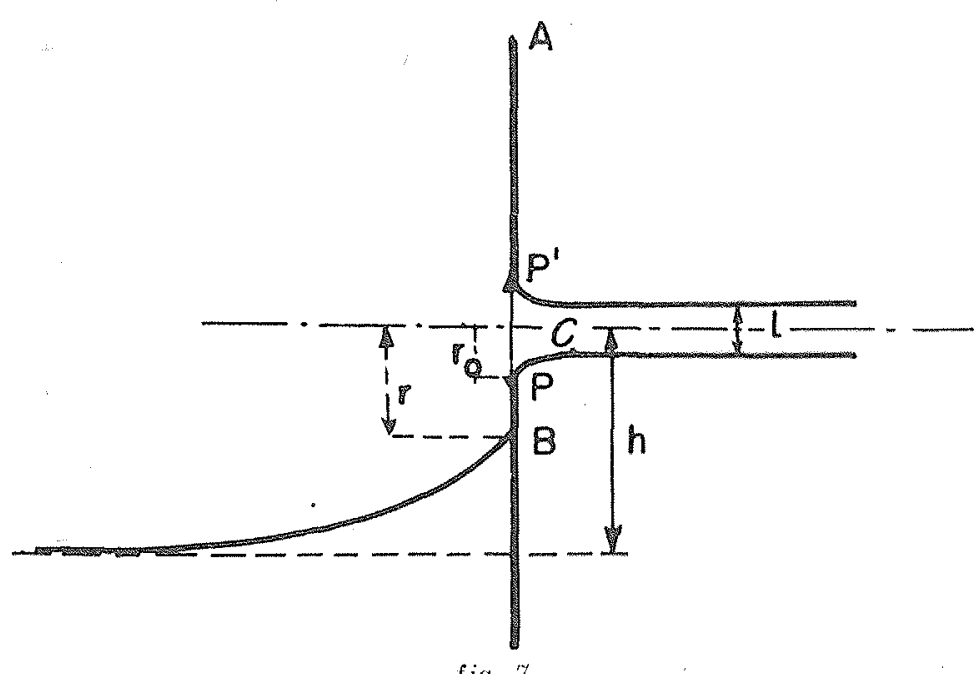

sont pas affectées sensiblement par la dimension de l'orifice, tant que la pointe $B$ de l'interface ne se rapproche pas trop de $P$, c'est-à-dire tant que la hauteur limite d'aspiration ne tombe pas ou-dessous de 3 ou 4 hauteurs de fente.

On peut aussi, à titre comparatif, effectuer un calcul tout analogue à celui que nous a donné la première estimation de la hauteur limite (paragraphe 3).

D'après ce schéma la position de B est donnée par

$$
\frac{v^{2}}{2}=\frac{\Delta \rho}{\rho} g(h-r)
$$

où $V$ doit être remplacé par ct et $r$ par son expression $7-1$.

Les conditions d'aspiration limite correspondent à l'existence pour cette équation d'une racine double, c'est-ò-dire à :

$$
\frac{\Delta \rho}{p} \frac{g r_{0}^{3}}{q^{2}}=\frac{(\pi+2)^{3}}{8 \pi^{2}} i^{3} \frac{1+t^{2}}{1-t^{2}}
$$

La relation $7-2$ donne alors compte tenu de $7-3$

$$
\frac{h}{r_{0}}=\frac{1}{\pi+2}\left[\frac{3}{t}-\frac{2 \phi}{1+t^{2}}+4 \operatorname{Arctg} t\right]
$$

Les deux relations $7-3$ et $7-4$ établissent sous forme paramétrique une relation entre $\frac{\Delta p}{p} g \frac{h^{3}}{q^{2}}$ et $\frac{h}{r_{0}}$ (en conformité avec les règles de l'Analyse Dimensionnelle); elles confirment h que le paramètre - intervint peu dès que sa valeur dépasse 6 ou 8

\section{CAS D'UN ECOULEMENT A TROIS DIMENSIONS}

Nous avons jusqu'ici porté notre attention principalement sur les écoulements plans.

Dans le cas d'un écoulement à trois dimensions nous avons moritré que les conditions d'aspiration limite s'expriment par :

$$
\Pi=\frac{\Delta p}{p} g \frac{h^{5}}{Q^{2}}=C^{\text {te }}
$$

II reste à évaluer la valeur de la constante, et nous n'avons ici d'autre ressource que la théorie élémentaire du paragraphe 3 .

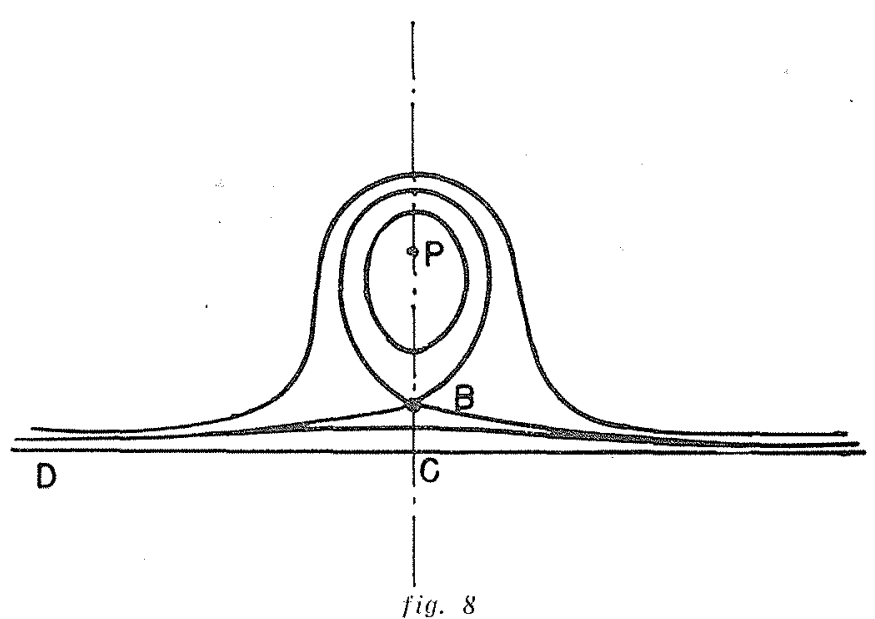

La figure 8 représente le plan vertical dans lequel est percé le petit orifice $P$; D est la trace du plan de l'interface à l'infini amont; à chaque point de contact $M$ de l'interface avec ce plan on a la relation fondamentale.

$$
\frac{V^{2}}{2}=\frac{\Delta \rho}{\rho} g(h-r \cos \rho)
$$

Nous admettrons encore que cette vitesse vérifie d'autre part la loi d'un puits ponctuel:

$$
V=\frac{Q}{2 \pi r^{2}}
$$

Les conditions d'aspiration limite sont évidemment réglées par la possibilité de trouver 
un point de raccordement de l'interface entre $P$ et $C$; celui-ci vérifie

$$
8 \pi^{2} \frac{\Delta \rho}{\rho} \frac{g h^{5}}{Q^{2}}(1-\rho) \rho^{4}=1 \text { oi } \rho=\frac{r}{h}
$$

et il cesse d'exister à partir du moment où cette équation a une racine double, c'est-à-dire pour :

$$
\Pi=\frac{\Delta \rho}{\rho} \frac{g h^{5}}{Q^{2}}=\left(\frac{5}{4}\right)^{5} \frac{1}{2 \pi^{2}}=0,154(8-3)
$$

Une représentation imagée de cette condition d'aspiration limite, consiste à suivre l'évolution des courbes de contact de l'interface donnée par $8-1$ et $8-2$ (fig. 8 ) ; la valeur 8 - 3 de 11 donne la courbe présentant un point double en $B$.

Nous avons déjà dit toutes les réserves que soulève dans le détail cette théorie élémentaire; cependant il se trouve ici encore qu'elle donne des résultats en remarquable accord avec l'expérience (voir communication de $M$. Paul GARIEL).

\section{INFLUENCE DE LA VISCOSITE}

- Dans tout ce qui précède nous avons négligé la viscosité du liquide; ce facteur est-il susceptible d'avoir une influence et dans quelle mesure; c'est ce que nous voudrions analyser maintenont.

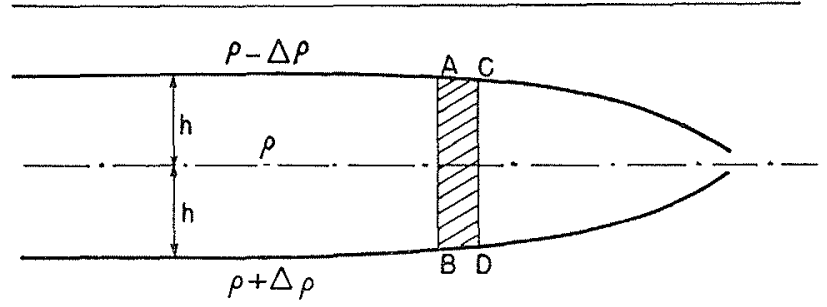

fig. 9

Nous considérerons pour cela un canal comportant trois couches de densité différente (fig. 9); une couche médiane de densité o disposée symétriquement de part et d'autre de la fente; au-dessus une nappe s'étendant jusqu'au niveau libre du canal de densité o- $\triangle 0$; au-dessous enfin une masse liquide de densité o $+\Delta 0$; nous supposons que le débit q aspiré par la fente intéresse uniquement la couche médiane, les deux autres demeurant rigoureusement au repos.

Il est à prévoir d'après les considérations du paragraphe 3, que l'écoulement du fleuve sousmarin doit être analogue à un écoulement fluvial ordinaire, à condition toutefois de remplacer lo pesanteur $g$ par une pesanteur réduite $\frac{\Delta \rho}{\rho} \mathrm{g}$.
Le phénomène de l'aspiration limite comporterait alors deux stades.

Un premier stade est déterminé au voisinage même de la paroi : il est dominé par les considérations dynamiques qui ont été développées précédemment (moyennant quelques modifications nécessitées par notre nouveau schéma).

A partir de là et en remontant vers l'amont le phénomène est en quelque sorte pris en main par le frottement visqueux ou turbulent; cet effet se cumulant avec la distance transforme l'interface presque horizontale du stade précédent en une ligne de remous incurvée.

Pour analyser cet effet nous nous placerons dans l'approximation du courant graduellement varié, c'est-à-dire courbure faible des filets et en conséquence répartition hydrostatique des pressions sur une verticale.

Le bilan des forces de pression agissant sur une tranche de courant de longueur $\Delta x$, de largeur unité (fig. 10) est représenté par l'aire de trapèze abcd.

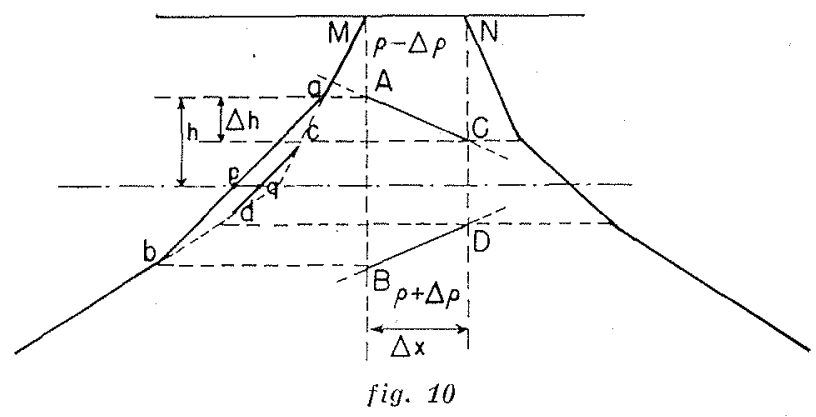

On a :

$$
\overline{p q}=g \Delta \underline{p} \Delta h
$$

et aire $a b c d=2 h \overline{p q}$

D'où une force résultante

$$
F_{1}=-2 h g \triangle p \triangle h
$$

D'autre part si nous appelons : la tension tangentielle aux interfaces la force de résistance s'écrit :

$$
F_{2}=-2 \tau \Delta x
$$

et I'on peut relier - à la vitesse moyenne $V$ par

$$
=\text { is } \mathrm{U}^{2}
$$

Ecrivant que ces deux forces donnent à lo dU tranche une accélération moyenne a $U \frac{}{d x}$ nous obtenons

$$
\frac{\Delta \rho}{\rho} g \frac{d h}{d x}+\alpha U \frac{d U}{d x}+\lambda \frac{U^{2}}{h}=0
$$


On reconnait l'équation du remous pour un canal horizontal où toutefois la pesanteur serait réduite à $\frac{\triangle \rho}{p} g$; il en résulte une beaucoup plus grande sensibilité de l'interface aux forces de friction, lesquelles sont d'ailleurs faibles en raison de la petitesse des vitesses d'écoulement.

Pour les régimes laminaires et turbulent-lisse. i. est fonction du nombre de Reynolds:

$$
R=4 \frac{\mathrm{Uh}}{y}=2 \frac{\mathrm{q}}{y}
$$

et il demeure constant tout le long du courant.

L'intégration de $9-1$ conduit alors à l'équation de la ligne de remous

$$
\frac{\Delta p}{p} g h^{4}-\alpha q^{2} h^{\circ}+\lambda q^{2} x=c+e
$$

ou encore en comptant l'abscisse $x^{\prime}$ en sens inverse du courant

$$
\frac{\Delta p}{\rho} \frac{g h^{3}}{q^{2}}=\alpha+\lambda \frac{\left(x^{\prime}-c\right)}{h}
$$

Le coefficient $\lambda$ est une fonction décroissante du nombre de Reynolds et son influence est donc le plus sensible aux petits débits et pour le régime laminaire; on a alors:

$$
i=\frac{6 U}{q} \quad \%=1,20
$$

Le rapprochement qui précède avec les lignes de remous naturelles conduit également à généraliser la notion de régime critique, qui correspond dans le cas présent à :

$$
U=\sqrt{g^{\prime} h} \quad g^{\prime}=\frac{\Delta p}{p} g
$$

c'est-à-dire

$$
\frac{\Delta p}{p} g \frac{h^{3}}{q^{3}}=\frac{1}{4}
$$

Le paramètrell caractérise donc aussi le facteur cinétique du fluide sous-marin, et si I'on extrapole les résultats antérieurs au cas actuel on voit que les conditions d'aspiration limite correspondent à un régime tranquille.

L'interprétation de $\sqrt{g^{\prime} h}$ comme célérité d'une petite onde de translation demeure également sous certaines réserves; elle explique les propagations d'onde d'interface au grand ralenti que révèle l'expérience.

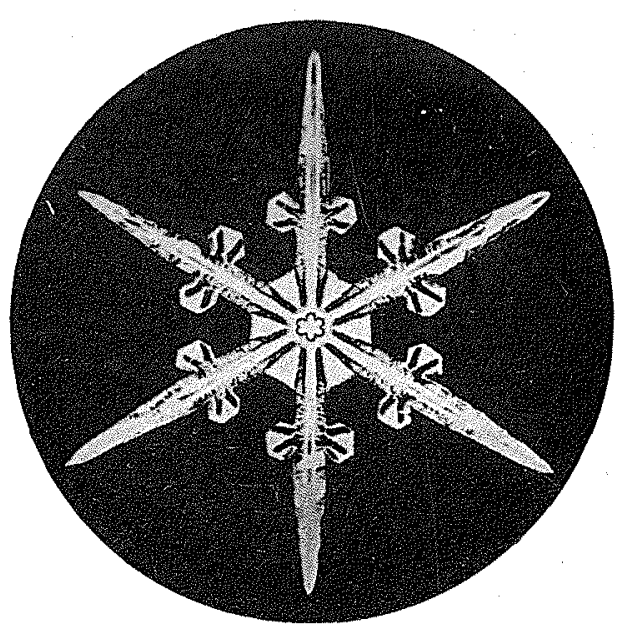

